\title{
Tangence
}

\section{Les archives du vent (2)}

\section{Jacinthe Martel, Benoît C. Gauthier, Sophie Marcotte et David Groulx}

Numéro 91, automne 2009

Le dire-monstre

URI : https://id.erudit.org/iderudit/044813ar

DOI : https://doi.org/10.7202/044813ar

Aller au sommaire du numéro

Éditeur(s)

Tangence

ISSN

1189-4563 (imprimé)

1710-0305 (numérique)

Découvrir la revue

Citer ce document

Martel, J., Gauthier, B. C., Marcotte, S. \& Groulx, D. (2009). Les archives du vent (2). Tangence, (91), 113-136. https://doi.org/10.7202/044813ar d'utilisation que vous pouvez consulter en ligne.

https://apropos.erudit.org/fr/usagers/politique-dutilisation/ 


\title{
Les archives du vent (2)
}

\author{
Jacinthe Martel, Université du Québec à Montréal, \\ et collaborateurs
}

Cette deuxième livraison de la chronique «Les archives du vent» propose les mêmes rubriques que la première, mais elle modifie sensiblement la teneur ou la portée de quelques-unes d'entre elles. S'il a d'abord semblé utile de présenter notre objet et d'expliquer le fonctionnement de cette chronique, il a aussi fallu, à l'été 2008, proposer une sorte de portrait général des travaux récents portant sur des fonds d'archives littéraires québécois et sur les différents modes et lieux de diffusion (expositions, numérisation, centres et équipes de recherche, etc.) mis en place par les deux principales institutions responsables de l'acquisition et de la mise en valeur des archives: Bibliothèque et Archives nationales du Québec (BAnQ) et Bibliothèque et Archives Canada (BAC). Comme les recensions présentées dans cette seconde chronique concernent une seule année, la première rubrique (Échos) est succincte; une place plus grande est ainsi faite aux autres. Dans les "Notes de recherche», Benoît C. Gauthier présente le contenu et analyse le potentiel de recherche du fonds André Brassard conservé à $\mathrm{BAC}$; ces documents permettent d'aborder l'apport du metteur en scène dans la création d'une pièce ${ }^{1}$. C'est un aperçu des travaux réalisés par Sophie Marcotte en vue de l'édition électronique de $\mathrm{La}$ détresse et l'enchantement (BAC) de Gabrielle Roy que propose la rubrique "Documents d'archives» qui contient par ailleurs, grâce à l'aimable collaboration de la succession Gabrielle Roy, un facsimilé et des transcriptions du manuscrit. Le résumé du mémoire de Gabrielle Demers, qui exploite en partie les documents du fonds Paul-Marie Lapointe (BAnQ) pour proposer des analyses génétiques de quelques poèmes du Vierge incendié, ainsi que les «Pistes bibliographiques» viennent clore l'ensemble. Outre les recensions des récents travaux portant sur les archives d'écrivains,

1. Dans «L'envers et l'endroit du texte de théâtre. La génétique au service de l'histoire» («Les archives du vent», Tangence, Rimouski et Trois-Rivières, $n^{\circ} 87$, été 2008, p. 96-102), Yves Jubinville a abordé la question d'une nouvelle histoire de la dramaturgie qui serait «fondée sur les pratiques d'écriture». 
cette bibliographie propose un inventaire sommaire des nouvelles acquisitions et des ajouts effectués dans des fonds déjà existants conservés dans diverses collections depuis 2005, année où paraissait un premier Répertoire des archives littéraires et des manuscrits d'écrivains ${ }^{2}$.

Sans fournir des descriptions qui soient aussi précises que celles que propose le Répertoire, cet inventaire en constitue pour ainsi dire une mise à jour et permet de constater de plus en plus l'existence d'un réel intérêt, de la part des écrivains eux-mêmes, non seulement pour la conservation de leurs archives, mais aussi pour leur intégration au sein des collections de différentes institutions. Les documents privés passent pour ainsi dire dans le domaine public et ce, avant même que l'œuvre d'un écrivain ne soit close. Les institutions mettent d'ailleurs en place divers mécanismes pour inciter les écrivains à déposer leurs archives ou à créer un fonds pour y verser périodiquement des documents. Chaque année, ce phénomène permet d'enrichir les collections de manière substantielle et d'ouvrir plus grand encore les portes de l'atelier des écrivains. Entre le travail du chercheur et le document d'archives, l'écart se réduit donc considérablement. Mais les écrivains sont parfois réticents à laisser l'entière gestion de leurs archives à celui ou à celle qui, plus qu'un intermédiaire entre le chercheur et l'accès à un fonds, constitue pourtant une sorte de guide; quand un archiviste dresse l'inventaire minutieux et précis d'un fonds, il en connaît tous les recoins et sait bien ce qui est susceptible d'intéresser les chercheurs. Le développement des collections ainsi que la réalisation d'inventaires précis, périodiquement mis à jour et disponibles en format électronique sont des atouts indéniables pour la recherche; le travail est déjà bien amorcé par les différentes institutions, mais il reste à souhaiter qu'elles puissent enrichir progressivement les collections numériques déjà existantes et que des inventaires numériques viennent suppléer l'absence, pour une grande quantité de fonds, de répertoires imprimés.

Les lieux de diffusion pour les travaux de recherche consacrés aux archives d'écrivains sont fort peu nombreux. La création de la

2. Jacinthe Martel (dir.), Répertoire des archives littéraires et des manuscrits d'écrivains, Québec, Éditions Nota bene, coll. "Convergences», 2005. Des fonds acquis avant 2005, mais inconnus au moment de la préparation du Répertoire, y sont également recensés. 
Revue de Bibliothèque et Archives nationales du Québec, qui a fait paraître son premier numéro en 2009, ainsi que l'intérêt grandissant des revues savantes et des éditeurs contribueront sans aucun doute à faire en sorte que le caractère encore marginal de ce type de travaux se modifie peu à peu. Les articles, ouvrages, communications et colloques recensés dans cette chronique témoignent en effet du caractère à la fois dynamique et diversifié des travaux consacrés partiellement ou entièrement aux archives des écrivains. En s'assurant de développer les collections et de faciliter l'accès aux fonds d'archives des écrivains, en mettant progressivement en place des réseaux de collaboration entre les archivistes et les chercheurs, les institutions favoriseront le développement de la recherche et, surtout, la formation des jeunes chercheurs, en particulier des étudiants universitaires.

\section{Échos}

Au cours de 2008-2009, BAnQ a présenté deux expositions d'envergure consacrées à des écrivains. La première soulignait le $25^{\mathrm{e}}$ anniversaire du décès d'Yves Thériault et le cinquantenaire de la parution du célèbre roman Agaguk. Tout en mettant l'accent sur la diversité de l'œuvre, l'exposition «Yves Thériault: le pari de l'écriture " proposait un parcours fort intéressant de la vie et de la carrière de Thériault, qui était ponctué de plusieurs volets, et dont rend compte un magnifique catalogue; un grand nombre de fac-similés et de documents, ainsi que la très belle mise en page et l'utilisation de pages de couleur faisant écho à la composition de l'exposition, font de ce catalogue un document indispensable pour quiconque s'intéresse à l'œuvre de Thériault ${ }^{3}$.

L'exposition "L'archipel poétique de Paul-Marie Lapointe», qui s'est mérité deux prix importants ${ }^{4}$, prenait place dans le cadre de la série intitulée "Ateliers d'écrivains"; outre des ouvrages de Lapointe, elle contenait un grand nombre de fac-similés de manuscrits conservés à $B A n Q$ ainsi que des carnets originaux

3. Des colloques ont également eu lieu dans le cadre de ces expositions; voir les "Pistes bibliographiques».

4. Le studio Paprika a remporté un grand prix Grafika dans la catégorie Design ainsi qu'un Pencil d'argent dans la catégorie «Environmental DesignCampaign »(One Show Design, New York) pour le concept graphique de l'exposition. 
provenant de la collection privée de l'auteur ${ }^{5}$. Cette exposition marquait le $60^{\mathrm{e}}$ anniversaire de la parution du premier recueil de Lapointe, Le vierge incendié, dont elle présentait le manuscrit; elle offrait, dans une scénographie remarquable, un extraordinaire accès à l'invention poétique de Lapointe. Malheureusement, aucun catalogue n'en conserve les traces.

Enfin, il importe de signaler l'inauguration, en novembre 2008, du Centre d'archives Gaston-Miron (CAGM) associé au Centre de recherche interuniversitaire sur la littérature et la culture québécoises (CRILCQ-Université de Montréal). Dirigé par Karim Larose, le CAGM rassemble une collection numérique de plusieurs milliers de documents radiophoniques et télévisuels (œuvres, entrevues d'écrivains, etc.), qui proviennent des archives de la Société Radio-Canada et qui sont répertoriés dans un catalogue fort utile et facile à consulter en ligne. Le CAGM, qui propose aux chercheurs l'accès à une collection d'une étonnante étendue, se propose ainsi de mettre en valeur et d'étudier les "archives radiophoniques et télévisuelles sur la littérature et la culture ${ }^{6} »$.

$1^{\text {er }}$ juin 2009

\section{Notes de recherche}

Conditions d'énonciation scénique: examen des archives d'André Brassard ${ }^{7}$

Benoît C. Gauthier, Université du Québec à Montréal

Alors qu'il poursuit ses études classiques au collège SainteMarie, André Brassard s'initie au jeu en suivant des cours d'art dramatique chez madame Jean-Louis Audet, d'autres chez Paul Buissonneau, Henri Norbert et Jacques Zouvi. À la même époque, il se présente, sans succès, aux auditions de la télévision de RadioCanada et à celles de l'École nationale de théâtre du Canada. Un peu comme ses prédécesseurs, Jean Gascon et Jean-Louis Roux,

5. Malheureusement, les conditions de conservation de certaines salles ne permettent pas d'exposer des originaux, car ils risqueraient d'être abîmés.

6. http://www.criq.umontreal.ca/. L'écoute des documents est réservée aux chercheurs associés au CAGM.

7. Ce texte reprend, en la développant, une communication présentée dans le cadre du Forum Pratiques et usages de l'archive littéraire, ACFAS, Université d'Ottawa, 13 mai 2009. 
c'est par le biais du jeu que Brassard en vient à la mise en scène. À la même époque, il dirige des ateliers de jeu destinés aux jeunes femmes de collèges privés; c'est alors qu'il s'intéresse aux classiques français. En 1965, avec Jean Archambault, Jacques Desnoyers et Rita Lafontaine, Brassard fonde le Mouvement contemporain où il met en scène les auteurs de l'avant-garde européenne, auteurs qui l'accompagneront au cours de ses nombreuses années de pratique théâtrale (Genet, Beckett, pour ne nommer que ceux-ci). Â l'âge de 22 ans, le succès des Belles-sours le propulse à l'avant-scène et, dès lors, le répertoire québécois oriente le choix de ses pièces (Tremblay, Ducharme, Dubé, Garneau, Loranger, Beaulieu, etc.).

Dès 1982, alors qu'il est nommé directeur artistique au Théâtre français du Centre national des Arts (CNA), Brassard revient au répertoire international qu'il avait fréquenté lors de sa formation au collège classique. C'est au CNA qu'il signe certaines de ses mises en scène les plus importantes: Périclès, Britannicus (1982), L'opéra de quat'sous (1984), Les Paravents (1987). Cependant, la dramaturgie québécoise continue de retenir son attention: La contre-nature de Chrysippe Tanguay, écologiste (1983), Albertine en cinq temps (1984, 1986, 1989), Le vrai monde? (1987), Les muses orphelines (1988), Les feluettes (1989), La charge de l'orignal épormyable (1989), etc. Au CNA, il dirige aussi certains ateliers destinés aux maîtres-interprètes. Bref, de 1965 à 2008, Brassard aura dirigé plus de cent trente mises en scène ${ }^{8}$.

Le fonds André Brassard (Bibliothèque et Archives du Canada: R11897) expose la pratique du metteur en scène ${ }^{9}$ dans au moins quatre ensembles de documents: 87 cahiers de notes de productions théâtrales et cinématographiques (1973 à 2000), trois

8. Ces informations proviennent de l'ouvrage de Michel Vaïs, Dictionnaire des artistes du théâtre québécois, Montréal, Cahiers de théâtre Jeu/Québec Amérique, 2008, p. 68.

9. Le fonds Brassard contient également des documents divers relatifs aux mises en scène au théâtre et au cinéma (programmes, photographies, dessins, coupures de presse, dactylogrammes, plans de mise en scène), mais aussi des textes de travaux d'étudiants de l'École nationale de théâtre du Canada, des textes d'auteurs divers, des dossiers-sujets (correspondance, procès-verbaux, revues de presse, demandes de subvention, cahiers de rapports de lectures) et des documents personnels (cahiers de comptabilité, agendas, etc.). À notre connaissance, le fonds renferme deux types de documents olographes, soit les cahiers de notes de productions théâtrales et cinématographiques et les cahiers d'ateliers de jeu. 
cahiers d'ateliers de jeu organisés par Brassard en 1981, le Journal de bord de la production des Paravents (1986-1987) et des témoins de relectures et réécritures assistées. La trentaine de cahiers que nous avons consultés (ceux des années 1980, dont les entrées sont datées) couvrent les moments de répétitions. On y trouve des notes éparses où s'entremêlent des indications destinées aux acteurs, des rendez-vous, des adresses, des croquis de décors, etc. Essentiellement, il s'agit ici d'une écriture de l'immédiat.

À cet effet, notons que le travail du metteur en scène comprend, règle générale, trois étapes. Il y a d'abord celle de la "documentation", où il se familiarise avec le texte dramatique et repère ses enjeux, puis celle de la "conception" où il établit les conditions d'énonciation scéniques du spectacle. Ces conditions d'énonciation constituent une notion fondamentale au théâtre, puisqu'il s'agit de la médiation entre le texte et la scène ${ }^{10}$, de la conduite du texte dramatique en une écriture scénique, étape cruciale où le metteur en scène "projette dans l'espace ce que le dramaturge inscrit dans le temps ${ }^{11} »$. C'est au cours de la phase de la «réalisation » que le metteur en scène assure, en présence de son équipe de créateurs, un travail de coordination et d'homogénéisation relativement à l'écriture scénique. Avant de débuter une production, Brassard a la réputation d'effectuer une excellente étude du texte dramatique et de situer clairement les prémisses d'énonciation. Aussi, avons-nous été surpris de constater l'absence de notes relevant de cette étape du travail dans les cahiers de production.

Dans un autre ordre d'idées, nous avons entrepris d'identifier les documents qui témoignent de cette sensibilité réciproque que Brassard entretient avec ses acteurs. Deux ensembles de documents retiennent l'attention du chercheur: les cahiers d'ateliers de jeu qu'organise Brassard en 1981 et le Journal de bord de la production des Paravents. Les archives renferment trois cahiers qui fournissent

10. Anne Ubersfeld écrit à cet effet: «la mise en scène peut [...] exhiber les conditions d'énonciation de la fiction avec leurs diverses composantes, ou au contraire les modifier, voire en substituer d'autres, pour changer le sens, ou inversement accroître la lisibilité pour un spectateur actuel, qui ne saisirait pas des conditions d'énonciation historiquement devenues obscures. C'est là que se fait l'une des tâches essentielles de la mise en scène» (Les termes de l'analyse du théâtre, Paris, Seuil, 1996, p. 37).

11. Nous reprenons ici les propos d'Adolphe Appia, Acteur, espace, lumière, Paris, Théâtre populaire, $\mathrm{n}^{\circ}$ 5, 1954, p. 38. 
des informations fort intéressantes relativement à des séances d'exploration qui visent à sonder le personnage ${ }^{12}$. Ces cahiers rendent compte des répertoires d'exercices ${ }^{13}$ que privilégie Brassard et qui servent à questionner la notion de personnage en présence d'acteurs-créateurs. Intitulés "Cahier de préparation à l'atelier de mai-juin", "Atelier Racine» (juillet-août) et "Atelier de travail $^{14} »$ (été-automne), ils s'adressent aux maîtres-interprètes. Le fonds renferme aussi un autre ensemble de documents qui relève encore une fois de cette réciprocité entre Brassard et ses acteurs ${ }^{15}$. Il s'agit du Journal de bord des Paravents (Jean Genet), une mégaproduction réalisée par le Centre national des Arts et le Théâtre du Nouveau Monde en 1986-1987. Réalisé par Claire Faubert ${ }^{16}$ (d'août 1986 à février 1987), ce Journal, subdivisé en quatre cahiers, renferme quatre cent seize pages; il s'agit d'un document qui relate de façon fidèle et détaillée le quotidien d'une production théâtrale. Faubert prend soin de noter la conception de la mise en scène que confie Brassard aux créateurs rassemblés lors de la première réunion de production, indices qui guident les conditions d'énonciation du spectacle. À même ce journal, on pénètre au cœur de la vision du metteur en scène; du même coup, on côtoie tous les artisans et on assiste à chacune des étapes de la production. Faubert y détaille les coupures de texte, note les dates et les heures de répétitions, explicite les sessions de discussion entre les créateurs, leurs questionnements, leurs décisions, les ateliers d'exploration avec les acteurs et les directives du metteur

12. «[L]es metteurs en scène pour lesquels la notion de personnage recouvre une réalité tangible [...] l'utilisent comme unité de travail réelle au cours des répétitions, incitant l'acteur à concevoir le personnage comme entité anthropomorphe dotée d'une psychologie, d'une gestualité et d'un univers qui lui sont propres» (Josette Féral, Mise en scène et jeu de l'acteur: entretiens, Montréal/Carnières, L’Espace du texte, Éditions Jeu/Éditions Lansman, 1997, t. 1, p. 19).

13. Dans son ouvrage, Claude Lapointe note que Brassard «propose des exercices puisés chez Brecht et chez Sonia Moore, formatrice américaine d'acteurs, qu'il adapte à l'œuvre à produire» (André Brassard. Stratégies de mise en scène, Montréal, VLB éditeur, 1990, p. 61).

14. Certains de ces cahiers renferment la liste des noms des comédiens, des notes de cours et des précisions sur les rôles attribués aux participants.

15. En réalité, ces documents proposent bien d'autres avenues, mais la relation de travail entre le metteur en scène et ses interprètes y est privilégiée.

16. Comédienne et metteure en scène, Claire Faubert fut l'une des interprètes de la compagnie permanente du Théâtre français au Centre national des Arts; elle fut aussi professeure (Université d'Ottawa) et directrice artistique (Théâtre du Trillium). 
en scène, réplique après réplique. La veille de la première, on trouve les notes du metteur en scène destinées aux acteurs. Faubert relève aussi les difficultés rencontrées par ces derniers avec leurs accessoires et leurs costumes. Enfin, elle prend soin de noter le déroulement des derniers enchaînements, les impressions de la première et des représentations subséquentes, de même que les impressions et propos du metteur en scène à la suite de la parution des critiques. La mise en scène a cette fâcheuse habitude de ne laisser aucune trace; aussi, ce journal est-il un document-témoin privilégié qui vise à confier à la mémoire les étapes et enjeux de celle-ci.

Nous remarquons enfin la présence de documents relatifs à cinq œuvres pour lesquelles Brassard s'engage avec un auteur dramatique dans un processus de relectures et de réécritures assistées. Nous retenons, chez Michel-Marc Bouchard, les versions III, IV et V du texte La contre-nature de Chrysippe Tanguay, écologiste (1983); le texte provisoire et la version finale des Feluettes (1985-1987); les versions I, II, III, IV et la version finale des Muses orphelines (mars-septembre 1988); enfin, chez Michel Tremblay, les versions II et III du Vrai monde? (1987) et les versions I et II de La maison suspendue (1989-1990). Les divers états de ces textes pourraient faire, tout au moins, l'objet d'études textologiques.

L'ensemble des documents contenus dans le fonds André Brassard présente un mérite génétique indéniable, mérite qui relève d'une considérable genèse interne et externe. On y retrouve en effet des documents proprement génétiques, tels les manuscrits de travail autographes du metteur en scène: cahiers, notes, plans, documents préparatoires; en outre, le dossier génétique est enrichi de documents qui fournissent des informations extérieures à la genèse, mais qui sont précieuses pour l'analyse: notes de productions, correspondance, documents visuels, programmes, coupures de presse, rapports de lectures, etc. Enfin, le fonds «porte les traces d'un acte, d'une énonciation en marche, d'une création en train de se faire, avec ses avancées et ses blocages, ses ajouts et ses biffures, ses pulsions débridées et ses reprises, ses relances et ses hésitations ${ }^{17}$ ».

17. Almuth Grésillon, Éléments de critique génétique, Paris, Presses universitaires de France, 1994, p. 33. 


\section{Documents d'archives}

\section{L'édition électronique de $L a$ détresse et l'enchantement de Gabrielle Roy}

Sophie Marcotte, Université Concordia, juin 2009

L'édition électronique de La détresse et l'enchantement, qui s'inscrit dans le cadre du projet "Gabrielle Roy: du manuscrit au virtuel ${ }^{18}$ ", marque la deuxième phase du vaste projet d'édition électronique des inédits et des manuscrits de Gabrielle Roy amorcé en 2002. Elle fait suite à l'édition du Temps qui m'a manqué, la troisième partie demeurée inachevée de l'autobiographie ${ }^{19}$, qui est disponible en ligne depuis janvier $2007^{20}$.

Le dossier de La détresse et l'enchantement est conservé dans deux collections d'archives. Les deux états manuscrits ainsi qu'un dactylogramme annoté (pour un total de 2267 feuillets) se trouvent dans le fonds Gabrielle Roy de Bibliothèque et Archives Canada, l'institution à laquelle Gabrielle Roy a remis la plus grande partie de ses archives littéraires et personnelles au début des années $1980^{21}$. Une autre portion du dossier appartient à la collection privée de François Ricard, qui a accompagné Gabrielle Roy dans la révision du texte et qui a préparé l'édition publiée à titre posthume en 1984. Il s'agit du dactylogramme annoté ayant servi à la publication de 1984, qui comporte des interventions manuscrites de Gabrielle Roy et de François Ricard. Ce dossier, qui contient 633 feuillets, renferme aussi quelques lettres adressées à F. Ricard dans lesquelles la romancière énumère des corrections qu'elle souhaite voir effectuer à son texte.

18. Ce projet de recherche, dirigé par Sophie Marcotte de l'Université Concordia et codirigé par Jane Everett et François Ricard de l'Université McGill, reçoit une subvention du Conseil de recherche en sciences humaines du Canada (2009-2012).

19. La version publiée en 1984 de La détresse et l'enchantement comporte deux parties: «Le Bal chez le Gouverneur» et «Un oiseau tombé sur le seuil». Gabrielle Roy avait prévu que son autobiographie serait divisée en quatre parties. Elle n'a eu le temps de compléter que les deux premières; la troisième a été laissée en chantier.

20. Voir http://gabrielle-roy.mcgill.ca/electronique-temps.htm (édition préparée par Sophie Marcotte, avec la collaboration d'Olivier Robert et d'Elizabeth Thompson pour la programmation). Le temps qui m'a manqué a été publié pour la première fois dans la collection «Cahiers Gabrielle Roy», aux Éditions du Boréal en 1997.

21. Numéros d'acquisition: MSS 1982-11 et MSS 1986-11. Voir http:// collectionscanada.gc.ca 
La séquence de travail est la suivante: (1) À la fin des années 1970, Gabrielle Roy rédige à la main deux états successifs des deux premières parties de La détresse et l'enchantement. (2) Elle fait ensuite dactylographier le second état de son manuscrit par une copiste. (3) Des corrections sont ensuite effectuées sur le dactylogramme, d'abord par Gabrielle Roy, ensuite par François Ricard. (4) À la suite de ces corrections, certaines pages, dactylographiées de nouveau ont remplacé les anciennes dans le dactylogramme ${ }^{22}$. L'essentiel du travail de correction et de réécriture effectué par G. Roy et F. Ricard a été réalisé en 1980-1981; ce travail a permis l'établissement du texte préparé par F. Ricard et publié de façon posthume en $1984^{23}$.

Le dactylogramme conservé à Bibliothèque et Archives Canada et celui qui se trouve dans la collection de François Ricard comportant des annotations différentes, il paraît évident que Gabrielle Roy a travaillé à la révision des deux états de manière simultanée, en ne reproduisant pas toutes ses corrections sur les deux copies. Cela pose évidemment problème pour la conception des modules de visualisation, dans la mesure où il devient impossible de replacer les différents dactylogrammes dans une séquence chronologique de rédaction.

On le constate depuis quelques années avec les projets d'envergure comme la mise en ligne de l'ensemble des manuscrits de Madame Bovary de Flaubert ${ }^{24}$, l'édition électronique devient un mode d'édition et de diffusion tout indiqué pour les dossiers génétiques et pour les textes inédits. Dans le cas de Gabrielle Roy, elle sert bien les textes qui ne font pas partie de l'œuvre canonique, comme les récits brefs, les nouvelles, les romans et la correspondance qui n'ont pas été publiés de son vivant ou dont elle n'a pas nécessairement souhaité la publication posthume ${ }^{25}$. L'édition électronique permet l'accès libre et gratuit aux manuscrits; elle

22. Les pages qui ont été remplacées se trouvent dans une chemise à part, dans la collection privée de François Ricard.

23. Gabrielle Roy, La détresse et l'enchantement, Montréal, Éditions du Boréal, 1984.

24. Voir: http://bovary.univ-rouen.fr

25. En plus de La détresse et l'enchantement, Gabrielle Roy avait exprimé le souhait que deux parties de sa correspondance soient publiées: les lettres à sa sœur Bernadette (Ma chère petite sour... Lettres à Bernadette 1943-1970, Montréal, Boréal, 1988) et les lettres à son mari, le docteur Marcel Carbotte (Mon cher grand fou... Lettres à Marcel Carbotte 1947-1979, Montréal, Boréal, coll. «Cahiers Gabrielle Roy», 2001). 
présente par ailleurs, grâce aux fonctionnalités techniques offertes par la plateforme informatique, l'ensemble des pièces des dossiers génétiques, et elle permet un relevé complet des variantes, qu'il est possible d'accompagner d'un appareil critique beaucoup plus volumineux (introduction, renvois intertextuels avec possibilité de lire les textes intégraux et non pas seulement la référence bibliographique, documents connexes tels correspondance et coupures de journaux, par exemple), puisque le support électronique n'impose pas les mêmes limites matérielles que le livre traditionnel ${ }^{26}$. Dans le cas du projet d'édition des manuscrits et des inédits de Gabrielle Roy, l'édition électronique ne vient pas remplacer l'édition traditionnelle; elle permet plutôt de la compléter et, du coup, de mieux marquer la différence fondamentale entre l'œuvre canonique de Roy et les manuscrits et inédits.

Nous reproduisons dans les pages qui suivent le feuillet qui inaugure le premier état manuscrit du «Bal chez le Gouverneur», la première partie de La détresse et l'enchantement, que nous faisons suivre de la transcription avec balises $\mathrm{XML}^{27}$, le langage employé pour la mise en page du texte sur support informatique ${ }^{28}$. Apparaissent ensuite la transcription nettoyée (c'est-à-dire dépouillée de tous les codes de transcription) de ce premier feuillet ${ }^{29}$ et la portion du texte publié en 1984 qui lui correspond.

26. On en trouvera un exemple dans l'édition électronique du Temps qui m'a manqué (http://gabrielle-roy.mcgill.ca/electronique-temps.htm), où nous avons pu reproduire de longs extraits de textes de Gabrielle Roy pour expliquer certains passages qui y renvoient de manière explicite ou implicite, notamment Rue Deschambault et La détresse et l'enchantement.

27. Le protocole établi rejoint les règles de l'initiative internationale de la Text Encoding Initiative (TEI). L'édition électronique de La détresse et l'enchantement est réalisée en collaboration avec le laboratoire NT2 de l'Université du Québec à Montréal.

28. La transcription en XML de la page manuscrite que nous reproduisons ici a été réalisée par Joëlle McKay-Dubois, étudiante à la maîtrise en littérature au Département d'études françaises de l'Université Concordia.

29. Il est interdit de reproduire l'image et sa transcription, en tout ou en partie. Les droits appartiennent à la succession littéraire de Gabrielle Roy, le Fonds Gabrielle Roy Inc. 


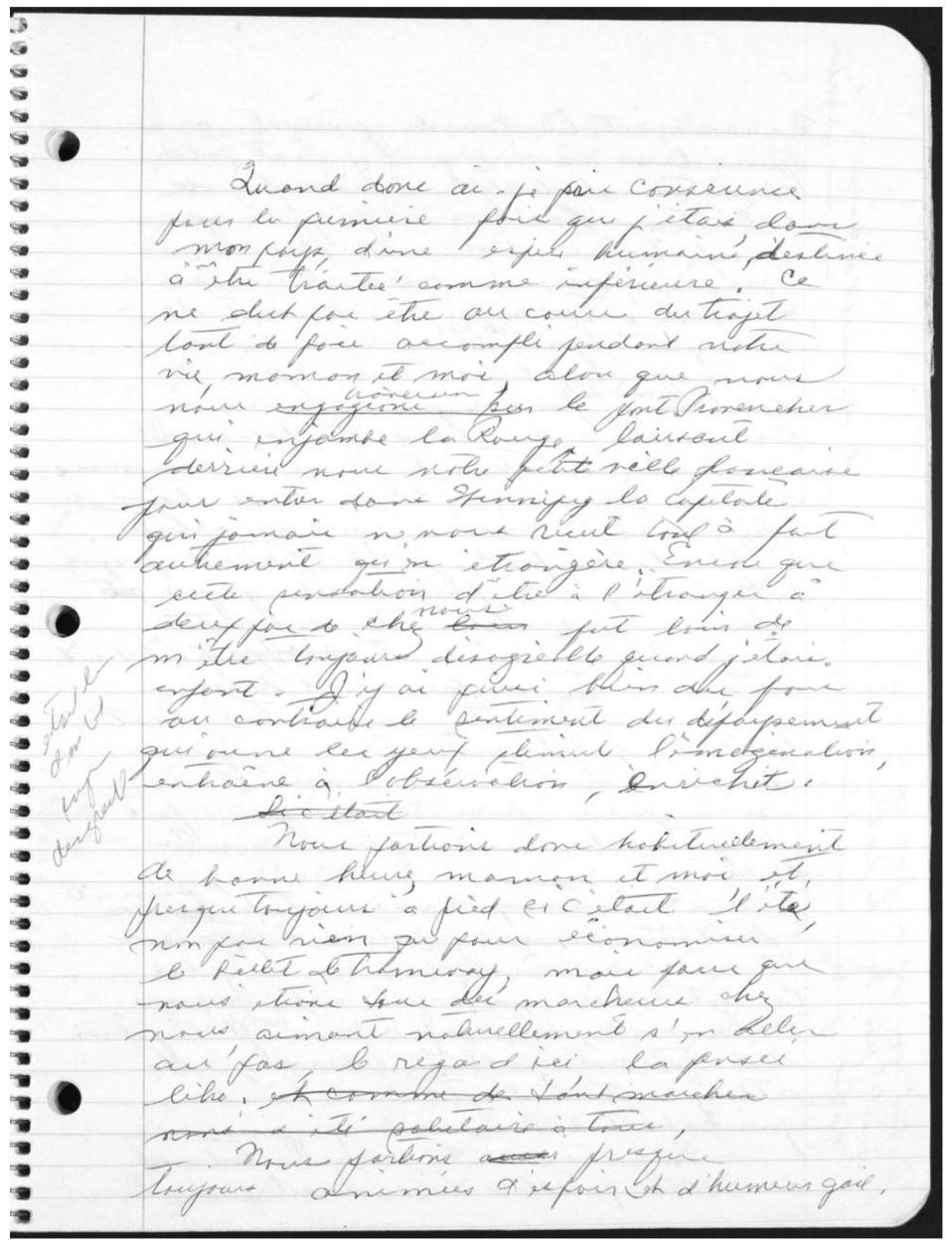


Transcription avec les balises XML

$<$ ?oxygen RNGSchema="http://www.tei-

c.org/release/xml/tei/custom/schema/relaxng/teilite.rng" type="xml"?>

- <TEI xmlns="http://www.tei-c.org/ns/1.0">

$-<$ teiHeader $>$

- <fileDesc>

- <titleStmt>

$<$ title>Détresse et Enchantement 002</title> $<$ titleStmt $>$

- <publicationStmt $>$

$<\mathrm{p}>$ Gabrielle Roy $</ \mathrm{p}>$

$<$ /publicationStmt $>$

- <sourceDesc $>$

$<$ p $>$ Joëlle Mckay-Dubois $</ p>$

$<$ sourceDesc $>$

$</$ fileDesc $>$

$</$ teiHeader $>30$

$-<$ text $>$

$-<$ body $>$

- $<$ p rend="indentfirst" $>31$

$<\mid \mathrm{b} />32$

Quand donc ai-je pris conscience

$<|\mathrm{b}|>$

pour la première fois que j'étais, dans

$<|\mathrm{b}|>$

mon pays, d'une espèce humaine destinée à $<|\mathrm{b}|>$

être traitée comme inférieure ! Ce $<\mathrm{lb} \mid>$

ne doit pas être au cours du trajet $<|\mathrm{b}|>$

tant de fois accompli pendant notre $<|\mathrm{b}|>$

vie, maman et moi, alors que nous $<|\mathrm{b}|>$

30. Les renseignements contenus dans cette portion de codes correspondent à l'en-tête du fichier. Ils ne sont pas impliqués dans la visualisation du texte qui découle de cette phase de balisage.

31. La balise $<\mathrm{p}>$ indique le début d'un paragraphe.

32. La balise $<\mathrm{lb}>$ indique un saut de ligne. 
nous

$<$ hi rend="ul" >engagions sur $</$ hi $>$

$<$ add place $=$ "interlinear" $>$ traversions $</$ add $>33$

le pont Provencher

$<\mid \mathrm{b} />$

qui enjambe la Rouge laissant $<|\mathrm{b}|>$

derrière nous notre petite ville française $<|\mathrm{b}|>$

pour entrer dans Winnipeg la capitale $<\mid \mathrm{b} />$

qui jamais ne nous reçut tout à fait

$<|\mathrm{b}|>$

autrement qu'en étrangère. Encore que

$<|\mathrm{b}|>$

cette sensation d'être à l'étranger à

$<\mid \mathrm{b} />$

deux pas de chez

$<$ add place $=$ "interlinear" $>$ nous $<$ /add $>$

$<$ del type="legible" rend="overstrike" $>$ |oin $</$ del $>{ }^{34}$

fut loin de

$<\mid \mathrm{b} />$

m'être toujours désagréable quand j'étais

$<\mathrm{lb} />$

enfant. J'y ai puisé bien des fois

$<\mathrm{lb} />$

au contraire le sentiment du dépaysement

$<\mid \mathrm{b} />$

qui ouvre les yeux, stimule l'imagination, $<\mid \mathrm{b} />$

entraîne à l'observation, enrichit.

$<|\mathrm{b}|>$

- <add place="margin-left" $>35$

était

<gap reason="illegible" resp="JM" />

[illis.]

33. La mention «interlinear» indique qu'une portion de texte a été ajoutée en interligne.

34. La mention «overstrike» signifie qu'une portion de texte a été inscrite pardessus une autre.

35. La mention «margin» signifie qu'une portion de texte a été inscrite en marge. 
$<$ /add $>$

$</ p>$

$-<p$ rend="indentfirst" $>$

$<|\mathrm{b}|>$

$<$ del type="legible" rend="overstrike" $>$ Si c'était $</$ del $>$

$</ p>$

$-<\mathrm{p}$ rend="indentfirst" $>$

$<|\mathrm{b}|>$

Nous partions donc habituellement $<\mathrm{lb} \mid>$

de bonne heure, maman et moi, et, $<|\mathrm{b}|>$

presque toujours à pied si c'était l'été, $<|\mathrm{b}|>$

non pas rien que pour économiser $<|\mathrm{b}|>$

le billet de tramway, mais parce que $<|\mathrm{b}|>$

nous étions tous des marcheurs chez $<\mid \mathrm{b} />$

nous, aimant naturellement s'en aller $<|\mathrm{b}|>$

au pas, le regard ici, la pensée $<|\mathrm{b}|>$

libre.

- <del type="legible" rend="overstrike"> et comme de tant marcher

$<|\mathrm{b}|>$

nous a été salutaire à tous,

$<$ /del $>$

$</ p>$

- $<$ p rend="indentfirst" $>$ $<|\mathrm{b}|>$

Nous partions

$<$ del type="legible" rend="overstrike" >aussi</del> presque $<|\mathrm{b}|>$

toujours animées d'espoir et d'humeur gaie. $</ p>$

$</$ body $>$

$</$ text $>$

$</$ TEl $>$ 


\section{Transcription nettoyée}

Quand donc ai-je pris conscience pour la première fois que j'étais, dans mon pays, d'une espèce humaine destinée à être traitée comme inférieure! Ce ne doit pas être au cours du trajet tant de fois accompli pendant notre vie, maman et moi, alors que nous nous ${ }^{36}$ traversions le pont Provencher qui enjambe la Rouge laissant derrière nous notre petite ville française pour entrer dans Winnipeg la capitale qui jamais ne nous reçut tout à fait autrement qu'en étrangère. Encore que cette sensation d'être à l'étranger à deux pas de chez nous fut loin de m'être toujours désagréable quand j'étais enfant. J'y ai puisé bien des fois au contraire le sentiment du dépaysement qui ouvre les yeux, stimule l'imagination, entraîne à l'observation, enrichit.

Nous partions donc habituellement de bonne heure, maman et moi, et, presque toujours à pied si c'était l'été, non pas rien que pour économiser le billet de tramway, mais parce que nous étions tous des marcheurs chez nous, aimant naturellement s'en aller au pas, le regard ici, la pensée libre. Nous partions presque toujours animées d'espoir et d'humeur gaie.

\section{Extrait du texte publié ${ }^{37}$}

Quand donc ai-je pris conscience pour la première fois que j'étais, dans mon pays, d'une espèce destinée à être traitée en inférieure? Ce ne fut peut-être pas, malgré tout, au cours du trajet que nous avons tant de fois accompli, maman et moi, alors que nous nous engagions sur le pont Provencher au dessus de la Rouge, laissant derrière nous notre petite ville française pour entrer dans Winnipeg, la capitale, qui jamais ne nous reçut tout à fait autrement qu'en étrangères. Cette sensation de dépaysement, de pénétrer, à deux pas seulement de chez nous, dans le lointain, m'était plutôt agréable, quand j'étais enfant. Je crois qu'elle m'ouvrait les yeux, stimulait mon imagination, m'entraînait à observer.

Nous partions habituellement de bonne heure, maman et moi, et à pied quand c'était l'été. Ce n'était pas seulement pour économiser mais parce que nous étions tous naturellement mar-

36. Le second "nous" est supprimé dans le deuxième état manuscrit.

37. Gabrielle Roy, La détresse et l'enchantement, Montréal, Boréal, coll. «Boréal compact», 1988, nº 7, p. 11. 
cheurs chez nous, aimant nous en aller au pas, le regard ici et là, l'esprit où il voulait, la pensée libre, et tels nous sommes encore, ceux d'entre nous qui restent en ce monde.

Nous partions presque toujours animées par un espoir et d'humeur gaie.

\section{Fiche signalétique}

Gabrielle Demers, «Matérialité du texte et picturalité poétique.

Étude du recueil Le vierge incendié de Paul-Marie Lapointe", mémoire de maîtrise, Montréal, Université du Québec à

Montréal, 2007, 102 p.

Ce document reprend intégralement le Résumé joint au mémoire.

L'objectif de ce mémoire est de développer une réflexion sur les relations texte-image en jeu dans l'œuvre de Paul-Marie Lapointe. À partir du recueil Le vierge incendié, il s'agit de démontrer l'évolution du traitement de l'espace de la page, actualisant une étude sémantique, thématique et visuelle. Notre travail a donc pour but l'élaboration d'une étude actuelle de cette ouvre de 1948, notamment de ses dimensions figurale et visuelle. Il convoque certaines théories littéraires (écriture fragmentaire, figure littéraire, lecture tabulaire), ainsi que des théories en arts visuels. Il touche ainsi aux questions de la poésie visuelle et des livres d'artistes, mais aussi, il permet une étude génétique des poèmes. Les questions d'espace-page et de poème-tableau sont aussi convoquées.

Nous posons l'hypothèse selon laquelle le poème permet, grâce à l'étude de ses qualités picturales et esthétiques, d'en élaborer une étude visuelle. Il s'agit de prolonger les analyses sémantique et thématique par ce lien indéniable qu'elles entretiennent avec le domaine visuel du texte poétique. En reliant les études déjà existantes (Haeck, Fisette et van Schendel), ainsi qu'en articulant une analyse exhaustive de la composition du recueil (Major, Melançon), tout en ralliant l'étude nouvelle des dimensions tant figurale (Lyotard, Gervais) que génétique (Martel, Maunet), Le vierge incendié de Paul-Marie Lapointe justifie la méthode utilisée pour aborder et confirmer cette hypothèse. En effet, c'est à partir de la rencontre et du dialogue des concepts littéraires (écriture fragmentaire: Blanchot, Michaud...), ainsi que de ceux de lecture tabulaire (Krüger, Vandendorpe) et des travaux concernant le poème-tableau (Maunet, Cornu), que s'élaborent les principaux 
postulats de notre mémoire. Ils permettent une lecture tout autre du travail de Lapointe, une lecture picturale et esthétique, motivée tant par l'interrelation entre la variété formelle du recueil et sa composition thématique, par la figure littéraire qui façonne le texte et par le poème-tableau.

Cette étude est novatrice en ce qu'elle offre des voies analytiques encore inexplorées. L'originalité du travail de recherche réside dans l'étude de nouveaux aspects théoriques et poétiques chez Lapointe (la figure littéraire, la dimension picturale et l'analyse génétique). Plutôt que de s'attarder uniquement au discours de révolte (souvent pointé chez Lapointe), les dimensions figurale, génétique et picturale rendent compte de la valeur et de la portée de ce recueil.

\section{Pistes bibliographiques}

David Groulx, Université du Québec à Montréal

\section{ARCHIVES D'ÉCRIVAINS : NOUVELLES ACQUISITIONS ET AJOUTS}

Aquin, Hubert (UQAm, 192P). Nouveau fonds acquis de Philippe et Stéphane Aquin. 0,85 m. de documents textuels, 5 documents photographiques. Se compose principalement de correspondances, de textes, de poèmes, de notes manuscrites, d'imprimés et de documents photographiques. Documents connexes: fonds Hubert Aquin (écrivain), 44P.

Aubert de Gaspé, Philippe (Centre de référence de l'Amérique française, collection du Fonds du séminaire de Québec, SME 13/MS-912). Nouveau fonds. Manuscrit partiel des Mémoires.

BeAulieu, Victor-Lévy (BAnQ, MSS-408 ${ }^{38}$ ). Ajout. 11.16 m. Contient diverses versions d'une centaine d'œuvres publiées ou inédites dans le domaine du roman, de l'essai, du théâtre, des émissions radiophoniques et télévisuelles, la correspondance de l'écrivain de 1965 à 2003, ainsi que des documents iconographiques et audiovisuels.

Beauchemin, Yves (BAnQ, MSS-460). Ajout. Comprend la correspondance récente et les dossiers professionnels, de

38. Étant donné le caractère récent de plusieurs ajouts à des fonds déjà existants, les cotes peuvent renvoyer aux fonds déjà constitués. 
nombreuses versions encore inconnues de son roman Le second violon, son journal intime et d'autres textes politiques et littéraires.

ChoquetTe, Gilbert (BAnQ, MSS-114). Nouveau fonds. 0,90 m. de documents textuels et d'autres documents.

Daveluy, Paule (BAnQ, MSS-477). Ajout. Plusieurs œuvres dans les domaines du roman, de l'écriture radiophonique ainsi qu'un complément à sa correspondance.

Dorion, Hélène (BAC, R12362-0-2-F). Nouveau fonds. 10,16 m. de documents textuels, 12 affiches, 35 photographies, 3 cassettes sonores, 17 disques compacts. Contient notamment des notes préparatoires et plusieurs états d'écriture de ses textes, des manuscrits de livres, des carnets et sa correspondance littéraire.

Gagnon, Madeleine (BAnQ, MSS-402). Ajout. Témoigne plus spécifiquement de l'écriture de ses derniers ouvrages: Le chant de la terre (2002), Je m'appelle Bosnia (2005) et Â l'ombre des mots (2007).

Harou, Lise (BAC, R11746-0-1-F). Nouveau fonds. Contient les brouillons, manuscrits et tapuscrits de toutes ses œuvres publiées, ainsi que des textes inédits et des manuscrits d'articles publiés dans des revues littéraires.

LAPOINTE, Gatien (Musée québécois de culture populaire, TroisRivières). Nouveau fonds. Contient des carnets, des notes, de la correspondance et un journal.

Malenfant, Paul Chanel (BAC, R12452-0-3-F). Nouveau fonds. 3,80 $\mathrm{m}$. de documents textuels, 2 affiches. Contient notamment des récits, des romans, des essais poétiques et de la correspondance, des manuscrits et des dactylogrammes.

MarCEL, Jean (BAC, R11774-0-1-F). Nouveau fonds. $80 \mathrm{~cm} \mathrm{de}$ documents textuels comprenant des manuscrits dactylographiés, avec corrections et ajouts autographes, et des notes de recherche pour des essais.

Miron, Gaston (BAnQ, MSS-410). Ajout. Quelques œuvres, volumineuse correspondance, plusieurs dossiers relatifs à sa vie littéraire.

Nelligan, Émile (BAnQ, MSS-82). Ajout. 14 pages détachées d'un carnet de textes en prose et en vers portant la signature du 
poète (vers 1935), ainsi que le manuscrit autographe du poème "L'ancolie» de Joséphin Soulary transcrit de mémoire par Nelligan.

OlLivier, Émile (Université de Montréal, P349). Nouveau fonds. $5,77 \mathrm{~m}$. de documents textuels et autres documents. Le fonds contient des manuscrits, plusieurs versions de ses romans, nouvelles et articles. Il renferme également de la correspondance, des notes manuscrites et un journal de voyage.

PILON, Jean-Guy (BAnQ, MSS-85). Ajout. Comprend des manuscrits de ses œuvres, des textes d'autres auteurs le concernant, une volumineuse correspondance, des dossiers et papiers personnels reflétant sa carrière.

SimARD, Jean (Université de Sherbrooke, P-52). Nouveau fonds. $1,05 \mathrm{~m}$. de documents textuels, 139 dessins, 282 photographies, 16 feuilles de musique et 13 cartes postales. Comprend des manuscrits et des tapuscrits, des synopsis d'émissions radiophoniques et sa correspondance.

VAC, Bertrand (pseudonyme d'Aimé Pelletier; BAnQ, MSS-52). Ajout. Comprend ses mémoires, sa correspondance et des documents iconographiques.

VAn SChendel, Michel (UQAM, 193P). Nouveau fonds. 10,22 m. de documents textuels et autres documents. Se compose principalement de correspondance, de textes, de poèmes, d'imprimés, de coupures de presse, de documents iconographiques et de documents photographiques.

Vigneault, Gilles (BAnQ, MSS-53, MSS-96). Ajout. Comprend environ 300 de ses chansons, une douzaine de dossiers de textes de poésie et de notes, des textes de la série télévisée Dans tous les cantons et d'autres émissions auxquelles il a participé.

\section{EXPOSITIONS}

«L'archipel poétique de Paul-Marie Lapointe», BAnQ, tenue à la Grande Bibliothèque du 21 octobre 2008 au 31 mai 2009. Responsables: Mariloue Sainte-Marie (commissaire d'exposition) et Pierre Nepveu (conseiller scientifique). Parallèlement, une "Journée d'échanges scientifiques autour de l'œuvre de Paul-Marie Lapointe» a été organisée par la BAnQ et le CRILCQ à la Grande Bibliothèque le 20 février 2009. 
"Yves Thériault: le pari de l'écriture», BAnQ, tenue à la Grande Bibliothèque du 23 septembre 2008 au 18 janvier 2009. Responsable: Renald Bérubé. Catalogue: Yves Thériault: le pari de l'invention, Québec, Bibliothèque et Archives nationales du Québec/Presses de l'Université Laval, 2008, $176 \mathrm{p}$.

\section{ÉDITIONS}

ANDRÈs, Bernard et Patricia WiLlEMIn-ANDRÈs, Journal du siège de Québec du 10 mai au 18 septembre 1759, Québec, Presses de l'Université Laval, coll. «L'Archive littéraire au Québec. Série Monuments », 2009, 259 p 39 .

\section{COLLOQUES ET COMMUNICATIONS ${ }^{40}$}

Colloque «Quinze ans dans l'œuvre d'Anne Hébert, 1958-1973», ACFAS, Université d'Ottawa, 13 mai 2009, organisé par Martin Doré (Université de Sherbrooke) et Patricia Godbout (Université de Sherbrooke).

- AnCrenat, Anne-Marie, «Kamouraska: du drame réel à l'œuvre».

- De Gaule, Christophe, "Le scénario de Kamouraska: adaptation et compromis».

Forum «Pratiques et usages de l'archive littéraire», ACFAS, Université d'Ottawa, 13 mai 2009, organisé par Yves Jubinville (UQAM), Jacinthe Martel (UQAM) et Jacques Paquin (UQTR).

- Gauthier, Benoît Charles (UQAM), «Le fonds d'archives André Brassard ou l'examen des conditions d'énonciation au théâtre ».

- Leblanc, Julie (Université de Toronto), «Les carnets inédits de Marie-Claire Blais».

39. Bernard Andrès et Pierre Monette, auteur de l'ouvrage Lettres d'un fermier américain (2009) publié dans la même collection, ont participé à une causerie intitulée "La Conquête: un événement historique controversé » qui était animée par Marc André Bernier (UQTR). Librairie Olivieri, le 3 juin 2009.

40. Seules les communications portant sur des questions d'archives ou d'éditions sont recensées ici. Les programmes complets sont disponibles sur les sites de BAnQ et de l'ACFAS. 
- Marcotte, Sophie (Université Concordia), «L'édition électronique des manuscrits de Gabrielle Roy».

- Montreuil, Sophie (BAnQ), "Les fonds d'archives privées: le défi de la diffusion à l'heure du numérique».

- Olscamp, Marcel (Université d'Ottawa), «La correspondance comme genèse: Jacques Ferron et les origines épistolaires du Ciel de Québec».

- Paquin, Jacques (UQTR), "Les proses de Gatien Lapointe: une écriture à huis clos?».

- Sainte-Marie, Mariloue (Université Laval et BAnQ), «Les lettres de Gaston Miron : les défis d'une édition critique».

Colloque international «Yves Thériault: écriture et imaginaire d'un conteur ", BAnQ et CRILCQ ; s'est tenu au Centre d'archives de Montréal de BAnQ, 17-18 novembre 2008.

- Bérubé, Renald (UQAR), "Richesse du fonds YvesThériault: inédits, correspondances et génétique littéraire».

- Destrempes, Hélène (Université de Moncton), «Regards croisés sur l'Amérique autochtone: une étude des relations entre Bernard Assiniwi et Yves Thériault».

- Lafrance, Hélène (Université Santa-Clara, États-Unis), "Les métamorphoses d'Agaguk: création, réception critique et adaptations".

- Montreuil, Sophie (BAnQ), "Les fonds d'archives privées de BAnQ: recherche et mise en valeur».

\section{MONOGRAPHIE ET DOSSIER}

Catalogue de la bibliothèque personnelle de Gaston Miron, Répertoire bibliographique et présentation de Marie-Andrée Beaudet, CRILCQ - Université de Montréal, coll. "Nouveaux Cahiers de recherche $7 », 2009,433$ p.

«Les écrivains québécois à la $\mathrm{BAnQ}$ ", À rayons ouverts, Montréal, automne 2008, $\mathrm{n}^{\circ} 77$, p. 5-33.

\section{OUVRAGE COLLECTIF}

Frenette, Yves (sous la dir. de), Le Centre de recherche en civilisation canadienne-française 1958-2008. Archives, recherche, diffusion, Ottawa, CRCCF/Le Nordir, 2008, 130 p. 


\section{ARTICLES ET CONTRIBUTIONS À DES OUVRAGES COLLECTIFS}

Gaulin, Michel, «Les Archives des lettres canadiennes", dans Yves Frenette (sous la dir. de), Le Centre de recherche en civilisation canadienne-française 1958-2008. Archives, recherche, diffusion, Ottawa, CRCCF/Le Nordir, 2008, p. 83-101.

Jubinville, Yves, «La stratégie du miroir. Rites génétiques dans Le ventriloque et Abraham Lincoln va au théâtre», dans David Gilbert (sous la dir. de), Le corps déjoué. Figures du théâtre de Larry Tremblay, Carnières-Morlanwelz, Lansman Éditeur, 2009, p. 101-110.

Jubinville, Yves, «Le partage des voix: approche génétique de la langue dans les dramaturgies québécoises contemporaines», Études françaises, Montréal, vol. 43, nº 1, 2007, p. 101-119.

LALONDE, Michel, "La collection de fonds d'archives du CRCCF: l'aventure du Canada français», dans Yves Frenette (sous la dir. de), Le Centre de recherche en civilisation canadiennefrançaise 1958-2008. Archives, recherche, diffusion, Ottawa, CRCCF/Le Nordir, 2008, p. 49-81.

LeBlanC, Julie, "Intertextualité et génétique textuelle», dans Catherine Viollet et Véronique Montémont (sous la dir. de), Intra-textualités, Louvain-La-Neuve, Academia Bruylant, 2009, p. 130-145.

Major, Robert et Alain Person, «La fabrique d'Agaguk», dans Renald Bérubé (dir.), Yves Thériault: le pari de l'écriture, Québec, Bibliothèque et Archives nationales du Québec/Presses de l'Université Laval, 2008, p. 96-110.

OlsCamp, Marcel, "Éditer un texte radiophonique: problèmes et défis du radioroman Le Survenant», Voix et Images, Montréal, vol. 33, no 3 (99), printemps-été 2008, p. 15-20.

Robert, Mario, "Chronique d'archives", Revue d'histoire de l'Amérique française, vol. 62, $\mathrm{n}^{\text {os }} 3-4$, hiver-printemps 2008, p. $609-616^{41}$.

SAINTE-MARIE, Mariloue, "Les écritures de Paul-Marie Lapointe», Revue de Bibliothèque et Archives nationales du Québec, Montréal, 2009, no 1, p. 114-117.

41. Cette chronique rend compte régulièrement des nouvelles acquisitions de différents centres d'archives à travers l'Amérique française. 
WATteyne, Nathalie, "Les lectures d'Anne Hébert », Les écrits, Montréal, août 2008, n 123, p. 53-73. 